\title{
Adverse Pregnancy Outcomes in Women with Sickle Cell Trait*
}

\author{
Whitney L. Wellenstein, MD ${ }^{1}$ Shannon Sullivan, MD ${ }^{1}$ Jeanne Darbinian, MS, $\mathrm{MPH}^{2}$ \\ Miranda L. Ritterman Weintraub, PhD, MPH $^{3}$ Mara Greenberg, MD ${ }^{1}$
}

1 Department of Obstetrics and Gynecology, Kaiser Permanente Medical Center, Oakland, California

2 Division of Research, Kaiser Permanente Medical Center, Oakland, California

${ }^{3}$ Department of Graduate Medical Education, Kaiser Permanente Medical Center, Oakland, California

\author{
Address for correspondence Whitney Wellenstein, MD, Department \\ of Obstetrics and Gynecology, Kaiser Permanente Medical Center, \\ 3600 Broadway, Oakland, CA 94611 \\ (e-mail: wwellenstein@gmail.com).
}

Am J Perinatol Rep 2019;9:e346-e352.

\author{
Abstract \\ Keywords \\ - adverse pregnancy \\ outcomes in African \\ American women \\ - hemoglobinopathy in \\ pregnancy \\ - sickle cell trait
}

Objective To compare adverse pregnancy outcomes between women with sickle cell trait (SCT) and women with normal hemoglobin.

Study Design A retrospective cohort study of women who delivered within Kaiser Permanente Northern California between 2006 and 2013. Using hemoglobin electrophoretic profiles, we defined women with hemoglobin AS (HbAS) as having SCT and those with hemoglobin AA (HbAA) as having normal hemoglobin. Outcomes were pregnancy-induced hypertension (PIH), small for gestational age (SGA), gestational diabetes (GDM), and preterm delivery (PTD). Demographic and pregnancy outcome variations were assessed in bivariate analyses. Multivariable logistic regression modeling was used to estimate odds ratios for the association between primary outcomes and selected characteristics.

Results Of 31,840 eligible women, 868 (2.7\%) had SCT. Women with SCT were more likely to have $\mathrm{PIH}(15.6 \%$ vs. $12.2 \%, \mathrm{p}$ value $=0.003)$ and SGA $(8.3 \%$ vs. $6.1 \%, \mathrm{p}$ value $=0.008)$, less likely to have GDM (6.8\% vs. $9.8 \%$, p value $=0.003)$ and had similar PTD prevalence $(8.1 \%$ vs. $7.6 \%$, p value $=0.600)$. In multivariable analyses, SCT was not an independent predictor of these outcomes. Racial/ethnic minorities had higher adjusted odds of PIH, SGA, and GDM.

Conclusion SCT alone does not appear to be associated with adverse pregnancy outcomes. Race/ethnicity is a risk factor for adverse pregnancy outcomes.

In the United States (US), African American women have been shown to have poorer obstetric outcomes than their white counterparts, including higher rates of preterm delivery, hypertensive disorders, diabetes, and intrauterine growth restriction. ${ }^{1}$ African American women also have a disproportionately higher likelihood than women of other races to have a diagnosis of sickle cell disease (SCD), which can lead to similar obstetrical

\footnotetext{
ePoster presentation: 64th annual ACOG Clinical and Scientific Meeting, Washington DC, May 13, 2016
}

received

March 8, 2019

accepted after revision

May 14, 2019
DOI https://doi.org/

$10.1055 / \mathrm{s}-0039-1695743$ ISSN 2157-6998. risks. Conversely, sickle cell trait (SCT) is generally considered to be a benign condition. SCT is estimated to affect three million people in the US. Among the African American population, 8\% carry a diagnosis of SCT. ${ }^{2}$

Sickle cell disease in pregnancy is associated with a six-fold increase in maternal mortality, and an elevated risk for pregnancy-induced hypertension (PIH), small-for-gestational-age birth weight (SGA), preterm delivery (PTD), and stillbirth. ${ }^{2}$ On the other hand, it is unclear if SCT independently confers elevated risk of adverse pregnancy outcomes, and the

Copyright @ 2019 by Thieme Medical Publishers, Inc., 333 Seventh Avenue, New York, NY 10001, USA Tel: +1(212) 584-4662.
License terms

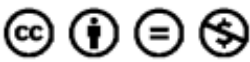


significance of SCT in pregnancy is centered on genetic screening to avoid or anticipate SCD in the offspring. ${ }^{3,4}$ However, it is possible that when under cardiovascular strain, SCT may contribute to physiologic changes that approach those seen in SCD, with resultant potential for increased morbidity and mortality. ${ }^{5,6}$ This phenomenon is well studied in athletes and army recruits where sudden death is increased under strained conditions. $^{6}$

Recent studies assessing the association between SCT and negative obstetric outcomes are mixed, with some studies finding a positive association with outcomes such as preeclampsia and preterm delivery, ${ }^{7}$ while other studies have found no significant association. ${ }^{4}$ In the studies that have seen no association between SCT and health outcomes, women with SCT have been analyzed along with women carrying other hemoglobinopathy traits that do not have the same potential for harm via a mechanism of vaso-occlusive disease. This theoretically may underestimate the true effect of SCT on obstetric outcomes and suggest that a more specific investigation of this area is warranted. ${ }^{4,7}$

Our study aimed to perform a robust analysis of a contemporary, racially, and ethnically diverse patient population with and without SCT to determine if the unique potential for vasoocclusion is independently associated with an elevated risk of adverse pregnancy outcomes. We hypothesized that SCT would impact the dynamic physiologic processes of pregnancy and therefore independently increase the risk of PIH, SGA, gestational diabetes mellitus (GDM), and PTD.

\section{Materials and Methods}

We conducted a retrospective cohort study of pregnant women who delivered between 2006 and 2013 within Kaiser Permanente Northern California (KPNC). KPNC is a large integrated health care delivery system, in which the insurance plan, inpatient and ambulatory services, and providers are organized under one network that provides all services to health plan members with few exceptions, such as referrals for highly specialized care. Women, therefore, receive prenatal care, testing, imaging, and delivery within one integrated system. The KPNC membership is similar to the wider insured population of the state of California, excluding those adults with very low income. ${ }^{8}$

All pregnant women in KPNC are offered an ethnicitybased hemoglobinopathy screening which recommends hemoglobin electrophoresis for women with a mean corpuscular volume $(\mathrm{MCV})<80 \mathrm{fL}$ or hemoglobin $<10.5 \mathrm{mg} / \mathrm{dL}$, and for those of Ashkenazi Jewish, Asian/Pacific Islander, or African American decent regardless of MCV. Women with a family history of hemoglobinopathy are offered screening. Women of Southeast Asian descent and African American descent are also recommended hemoglobin electrophoresis due to the increased risk of hemoglobin E and SCD. Women can also elect for screening without meeting ethnicity criteria.

Study data were collected using KPNC electronic medical records (EMR). From the KPNC Gestational Diabetes Pregnancy Registry, a large registry that identifies all pregnancies resulting in a live birth or stillbirth, we identified pregnan- cies within the health plan that continued past 20 weeks gestation. If there were multiple pregnancies for a woman in the stated time period, only the first pregnancy was selected. Women with a hemoglobin electrophoresis of AA (HbAA) or AS (HbAS) were included in the analysis. Any other electrophoretic profile was excluded. Women with a diagnosis of SCT were identified by an ICD-9 code of 282.5 linked to clinical encounters and by their hemoglobin electrophoretic profile. Patients with HbAS were defined as having SCT, and women with HbAA were defined as having a normal hemoglobin profile. Women whose pregnancy ended in a stillbirth and those with multiple gestations, history of renal disease, pre-existing diabetes mellitus, unknown GDM status, or missing or invalid data for infant birth weight or gestational age at delivery were excluded.

The primary outcomes of interest included disorders on the PIH spectrum (hereafter referred to as PIH) and SGA. We defined PIH as including gestational hypertension, superimposed pre-eclampsia, pre-eclampsia, eclampsia, and postpartum pre-eclampsia. PIH diagnoses were identified by ICD9 diagnosis codes alone. SGA was defined as birth weight less than $10 \%$ adjusted for gestational age based on the Fenton curves. Mode of delivery, birth weight and gestational age at delivery, and maternal demographic and other clinical characteristics such as medical comorbidites were obtained from infant and maternal electronic medical records.

Secondary outcomes were GDM and PTD. GDM was diagnosed by Carpenter-Coustan criteria: two or more abnormal values with a 100-g, 3-hour glucose tolerance test (fasting $\geq 95 \mathrm{mg} / \mathrm{dL}, 1$ hour $\geq 180 \mathrm{mg} / \mathrm{dL}, 2$ hour $\geq 155 \mathrm{mg} / \mathrm{dL}$, and 3 hour $\geq 140 \mathrm{mg} / \mathrm{dL}$ ) or a clinical encounter-linked diagnosis of GDM and a blood glucose level of $\geq 180 \mathrm{mg} / \mathrm{dL}$ measured one hour after a 50-g oral challenge. ${ }^{9}$ PTD was defined as delivery prior to 37 weeks gestation.

From previously published literature, we estimated that $\sim 15 \%$ of all pregnancies would result in one or more of adverse outcomes including PIH, SGA, or PTD. ${ }^{3}$ We hypothesized a 33\% increase in risk of adverse outcomes to $20 \%$ for women with HbAS compared with the baseline rate of $15 \%$ for those with HbAA. Using the proportions of 0.025 for the ratio of women in HbAS:HbAA (from feasibility estimates), we performed a twogroup continuity corrected chi-square test with a 0.05 twosided significance level to compare the groups. These results suggested that we would be able to detect the difference between $15 \%$ and $20 \%$ risk of adverse outcomes (odds ratio [OR] of 1.42 ) at $80 \%$ power with a minimum of 18,481 women in the HbAA group and 463 women in the HbAS group.

Descriptive statistics, including means and standard deviations for continuous variables and counts and percentages for categorical variables were used to summarize our data. We performed bivariate analyses using chi-square and Fisher's exact tests and $t$-tests and nonparametric Wilcoxon twosample tests for continuous variables. Logistic regression was used to estimate ORs and 95\% confidence intervals (CIs). Models were developed to determine if having SCT compared with a normal Hgb electrophoretic profile was associated with each outcome of interest, after controlling for potential confounders. We used a modified step-wise approach, 
starting with only the principal exposure (SCT vs. comparison group), and then sequentially adding covariates, which were associated with the principal exposure and/or outcome or were clinically relevant. The final model included age, race/ethnicity, pre-existing hypertension, pregravid BMI, and maternal level of educational attainment. All analyses were conducted using SAS 9.3 (SAS Institute, Inc., Cary, North Carolina). The KPNC institutional review board approved this study with waiver of written consent.

\section{Results}

Between 2006 and 2013, 216,530 women delivered within KPNC. The final cohort consisted of 31,840 women of which 868 (2.7\%) had SCT ( - Fig. 1). The demographic characteristics of the entire cohort and by study group are summarized in - Table 1. Compared with women with normal hemoglobin, subjects with SCT were more likely to be African American, and less likely to be white, Hispanic, and Asian/Pacific Islander. The SCT group was also younger and had higher BMI, less education, and more pre-existing hypertension. There were no differences in mode of delivery or gestational age at delivery between the SCT and comparison groups.

In bivariate analyses (-Table 2), women with SCT compared with those who had normal hemoglobin profiles were significantly more likely to have PIH and SGA and less likely to have GDM. The proportion with PTD was similar in both groups (8.1\% and $7.6 \%$, respectively, $p=0.61)$ and, therefore, PTD was not assessed in logistic regression modeling.

In bivariate analyses examining associations between other maternal characteristics and adverse pregnancy outcomes (-Table 2), women who had PIH compared with those who did not were older, more likely to be overweight or obese, African American or non-Hispanic white, and to have had pre-existing hypertension, and less likely to have graduated from college. Women who delivered an SGA infant were younger, less overweight or obese, and less likely to be nonHispanic white, more likely to be African American or Asian/ Pacific Islander, and to have had hypertension, compared with women whose infants were average- or large-for-gestational age. In contrast with women who did not have GDM, women with GDM were older, more likely to be Asian/Pacific Islander, African American, or Hispanic, overweight or obese, and to have had hypertension, and were better educated. Overall, there were statistically significant differences for age, race/ethnicity, pre-existing hypertension, and pregravid BMI between women with and without each of the outcomes.

In multivariable logistic regression analysis (-Table $\mathbf{3}$ ), there was no independent association between SCT and any

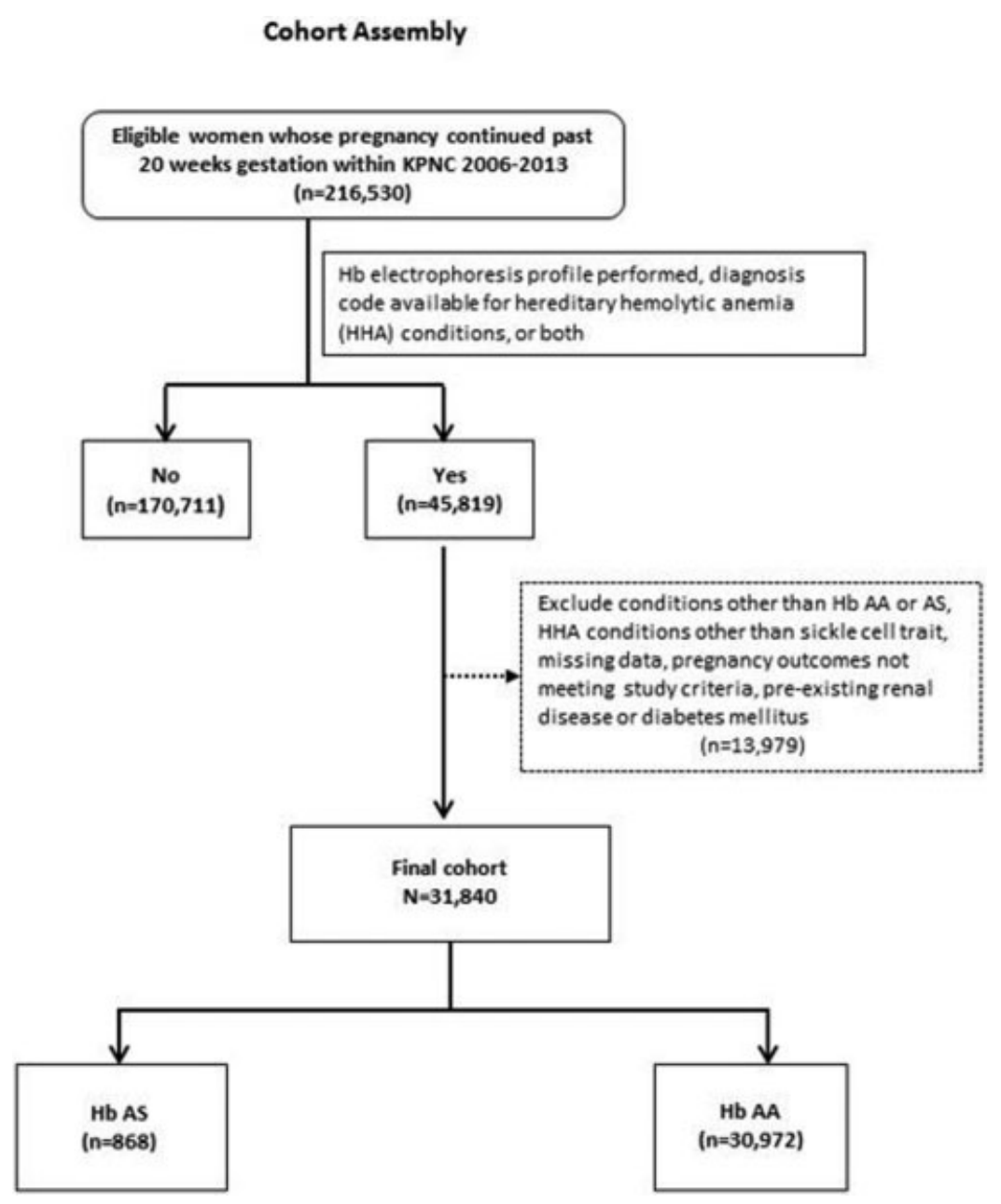

Fig. 1 Cohort selection. Flowsheet depicting cohort selection using inclusion and exclusion criteria. 
Table 1 Variations in demographic, clinical, and adverse pregnancy outcome characteristics according to sickle cell trait status

\begin{tabular}{|c|c|c|c|c|}
\hline & \multirow[b]{2}{*}{ Total $(n=31,840)$} & \multicolumn{2}{|l|}{ Sickle cell trait } & \multirow[b]{2}{*}{$p$ value } \\
\hline & & Yes $(n=868)$ & No $(n=30,972)$ & \\
\hline \multicolumn{5}{|l|}{ Demographic characteristics } \\
\hline Maternal age at delivery (years), mean (SD) & $29.2(6.2)$ & $27.3(6.6)$ & $29.3(6.2)$ & $<0.0001$ \\
\hline Maternal age at delivery (years), n (\%) & & & & $<0.0001$ \\
\hline$<20$ & $2568(8.07)$ & $114(12.13)$ & $2454(7.92)$ & \\
\hline $20-24$ & $4935(15.50)$ & $212(24.42)$ & $4723(15.25)$ & \\
\hline $25-29$ & $8350(26.22)$ & $220(25.35)$ & $8130(26.25)$ & \\
\hline $30-34$ & $9321(29.27)$ & $191(22.00)$ & $9130(29.48)$ & \\
\hline $35-39$ & $5272(16.56)$ & $94(10.83)$ & $5178(16.72)$ & \\
\hline $40+$ & $1394(4.38)$ & $37(4.26)$ & $1357(4.38)$ & \\
\hline Race/ethnicity, n (\%) & & & & $<0.0001$ \\
\hline African American & $8684(27.27)$ & $669(77.07)$ & $8015(25.88)$ & \\
\hline White & 4390 (13.79) & $25(2.88)$ & 4365 (14.09) & \\
\hline Hispanic & $5175(16.25)$ & $96(11.06)$ & $5079(16.40)$ & \\
\hline Asian/Pacific Islander (PI) & 11235 (35.29) & $15(1.73)$ & $11220(36.23)$ & \\
\hline Other & $2356(7.40)$ & $63(7.26)$ & $2293(7.40)$ & \\
\hline Pre-existing hypertension & $1511(4.75)$ & $63(7.26)$ & $1448(4.68)$ & 0.0004 \\
\hline Educational attainment level, n (\%) & & & & $<0.0001$ \\
\hline$<$ High school graduate & $2014(6.33)$ & $63(7.26)$ & $1951(6.30)$ & \\
\hline High school graduate & 4392 (13.79) & $135(15.55)$ & $4257(13.74)$ & \\
\hline Some college & $8830(27.73)$ & $294(33.87)$ & $8536(27.56)$ & \\
\hline College graduate or higher & $10609(33.32)$ & $199(22.93)$ & $10410(33.61)$ & \\
\hline Missing & $5995(18.83)$ & $177(20.39)$ & $5818(18.78)$ & \\
\hline \multicolumn{5}{|l|}{ Clinical characteristics } \\
\hline Body mass index & & & & $<0.0001$ \\
\hline$<25.0$ & $14799(46.48)$ & $299(34.45)$ & $14500(46.82)$ & \\
\hline $25.0-29.9$ & $7960(25.00)$ & $240(27.65)$ & $7720(24.93)$ & \\
\hline$>30$ & $7682(24.13)$ & $280(32.26)$ & $7402(23.90)$ & \\
\hline Missing & $1399(4.39)$ & $49(5.65)$ & $1350(4.36)$ & \\
\hline Gestational age at delivery, median (IQR) & $39.0(38.0-40.0)$ & $39.0(38.0-40.0)$ & $39.0(38.0-40.0)$ & 0.9400 \\
\hline Mode of delivery & & & & 0.0786 \\
\hline Vaginal delivery & $20923(65.71)$ & $591(68.09)$ & $20332(65.65)$ & \\
\hline Operative delivery & $1983(6.23)$ & $38(4.38)$ & $1945(6.28)$ & \\
\hline Cesarean delivery & $8871(27.86)$ & $236(27.19)$ & $8635(27.88)$ & \\
\hline Unknown & $63(0.20)$ & $3(0.35)$ & $60(0.19)$ & \\
\hline \multicolumn{5}{|l|}{ Adverse pregnancy outcomes } \\
\hline Pregnancy-induced hypertension & $3923(12.32)$ & $135(15.55)$ & $3788(12.23)$ & 0.0033 \\
\hline Small for gestational age & $1958(6.15)$ & $72(8.29)$ & 1886 (6.09) & 0.0076 \\
\hline Gestational diabetes & $3091(9.71)$ & $59(6.80)$ & $3032(9.78)$ & 0.0033 \\
\hline Preterm delivery & $2424(7.61)$ & $70(8.06)$ & $2354(7.60)$ & 0.6111 \\
\hline
\end{tabular}

Abbreviations: SD, standard deviation, IQR, interquartile range. 


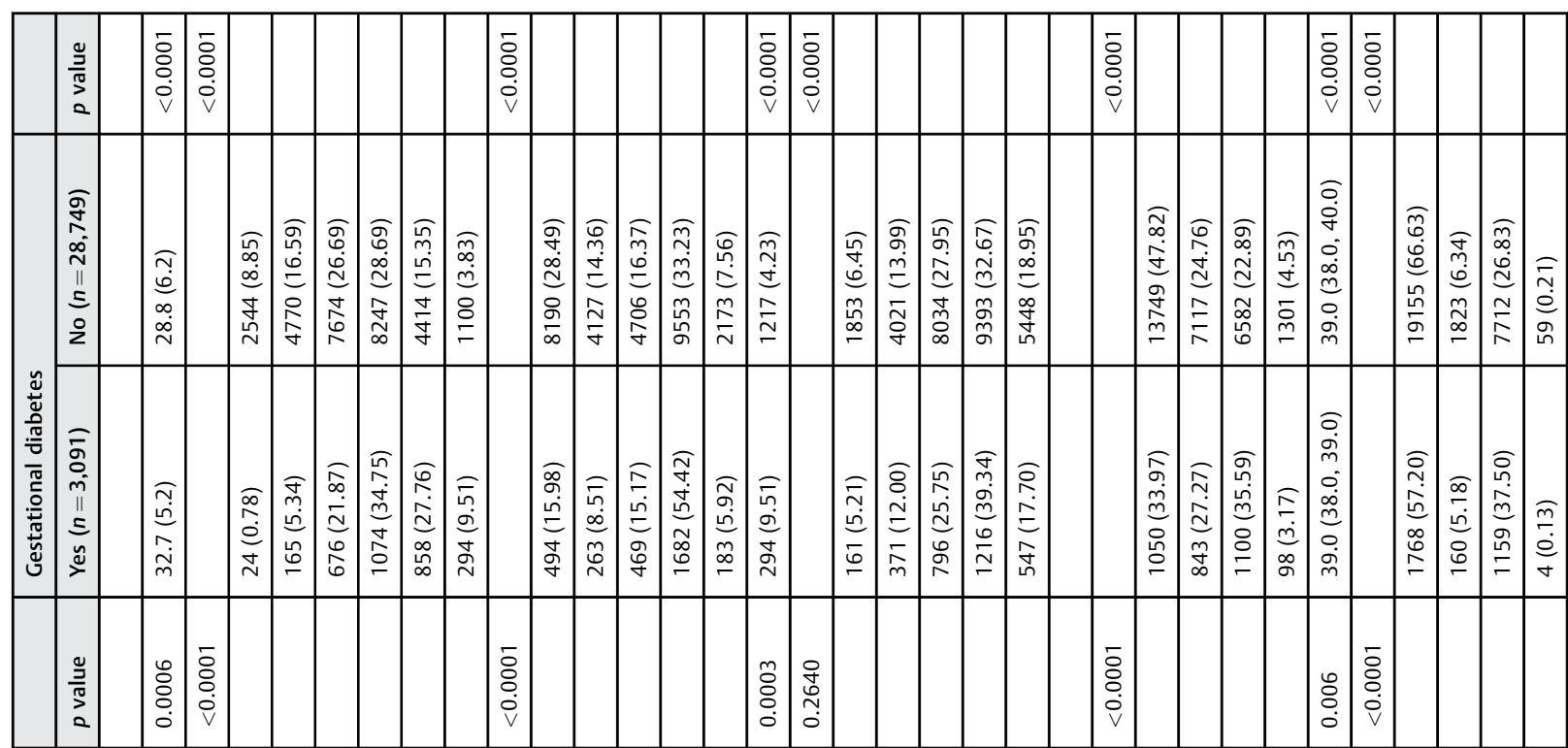

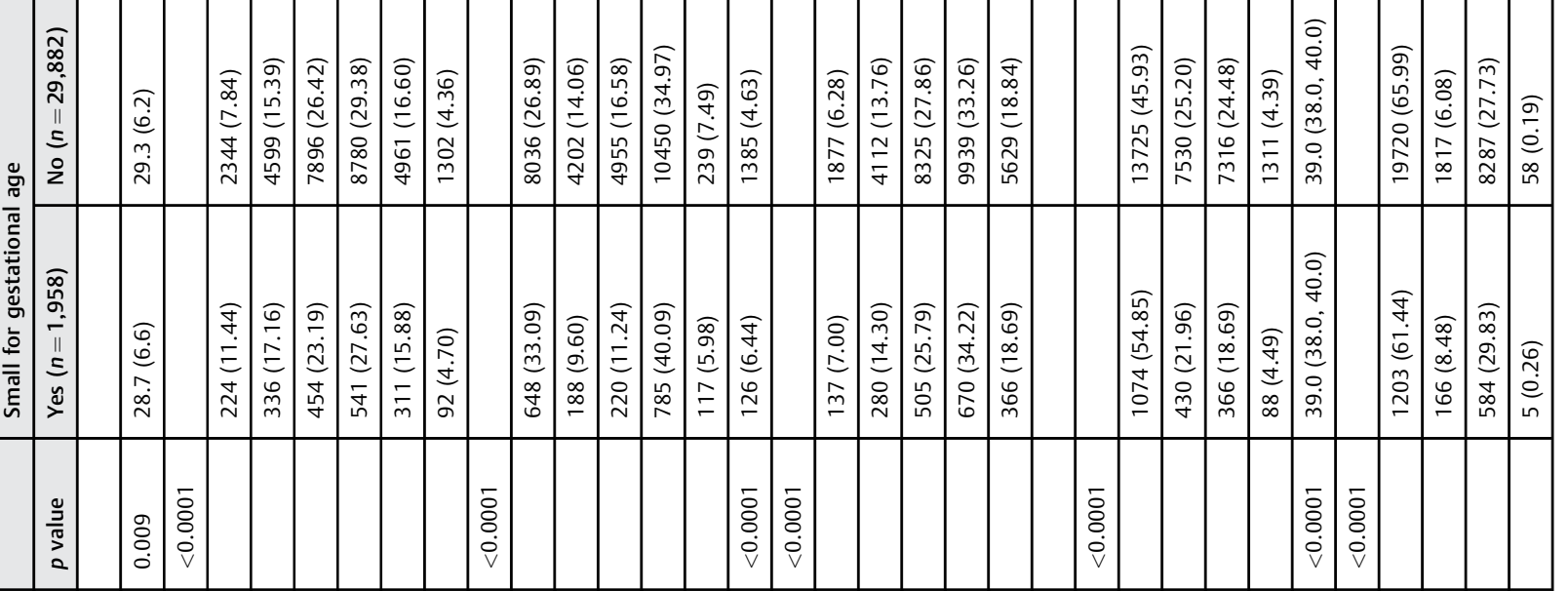


Table 3 Multivariable logistic regression of characteristics associated with adverse pregnancy outcomes, odds ratios (95\% confidence intervals) $(n=31,840)$

\begin{tabular}{|l|l|l|l|}
\hline & Pregnancy-induced hypertension & Small for gestational age & Gestational diabetes \\
\hline SCT (reference =no; Hb AA) & $0.98(0.80-1.20)$ & $1.24(0.97-1.60)$ & $1.17(0.89-1.56)$ \\
\hline Maternal age (continuous) & $0.99(0.99-1.00)$ & $0.99(0.98-0.99)$ & $1.11(1.10-1.11)$ \\
\hline Race/ethnicity (reference $=$ white) & & & \\
\hline African American & $1.05(0.94-1.17)$ & $1.77(1.49-2.09)$ & $0.93(0.79-1.09)$ \\
\hline Asian/Pacific Islander & $0.73(0.65-0.82)$ & $1.58(1.34-1.87)$ & $3.61(3.14-4.17)$ \\
\hline Hispanic & $0.80(0.71-0.91)$ & $0.99(0.81-1.21)$ & $1.58(1.34-1.86)$ \\
\hline Other & $0.87(0.74-1.01)$ & $1.13(0.89-1.43)$ & $1.48(1.21-1.81)$ \\
\hline Hypertension (reference $=$ no) & $10.10(9.00-11.32)$ & $1.70(1.40-2.07)$ & $1.49(1.28-1.72)$ \\
\hline BMI (reference $<25.0)$ & & & \\
\hline 25.0-29.9 & $1.58(1.44-1.73)$ & $0.74(0.65-0.83)$ & $1.91(1.73-2.12)$ \\
\hline$\geq 30$ & $2.28(2.08-2.50)$ & $0.62(0.55-0.71)$ & $3.50(3.15-3.88)$ \\
\hline Missing & $1.28(1.07-1.55)$ & $0.82(0.65-1.03)$ & $1.21(0.97-1.51)$ \\
\hline Educational $\geq$ college graduate) & & & $1.07(0.87-1.33)$ \\
\hline$<$ High school graduate & $0.97(0.82-1.15)$ & $1.02(0.87-1.19)$ & $1.43(1.18-1.72)$ \\
\hline High school graduate & $0.99(0.88-1.12)$ & $0.93(0.82-1.05)$ & $1.22(1.07-1.39)$ \\
\hline Some college & $1.03(0.93-1.13)$ & $0.95(0.83-1.09)$ & $1.08(0.97-1.19)$ \\
\hline Missing & $1.05(1.00-1.16)$ & & $1.02(0.91-1.14)$ \\
\hline
\end{tabular}

All three analyses mutually control for all listed covariates.

of the adverse pregnancy outcomes that were examined. BMI, race/ethnicity, and education were found to be independent risk factors.

\section{Comment}

Our findings suggest that SCT does not appear to be independently associated with PIH, SGA birth weight, or GDM. Compared with non-Hispanic whites, African American women had a disproportionately higher risk of PIH and SGA, Asian/Pacific Islander women were also at elevated risk of having SGA and GDM, and Hispanic women were at increased risk of GDM. A greater proportion of African American women and those with pre-existing hypertension accounted for the variations in PIH and SGA prevalence.

Our data are consistent with current literature highlighting racial and ethnic disparities in obstetrical outcomes. Our study revealed that even in an integrated health care system, race and ethnicity, specifically African American and Asian race, continue to be associated with adverse pregnancyrelated outcomes. The increase in adverse pregnancy outcomes experienced by African American women in our study does support findings from studies conducted throughout the 1960 s and $1970 \mathrm{~s}^{10}$ that found a positive relationship between PIH and SCT. However, these studies did not take into account race/ethnicity and did not examine hemoglobin electrophoresis. More recent studies that have assessed hemoglobin $C$ carriers, ${ }^{3}$ in addition to those with SCT, did not show increased maternal or fetal adverse outcomes associated with maternal SCT or hemoglobin C trait. However, Hemoglobin $C$ does not have the potential for vaso- occlusion nor does it have the same hypothetical vascular compromise as SCT. Therefore, we sought to examine the potential effects of SCT in isolation from other conditions of altered hemoglobin. Based on our results, we cannot consider SCT to be an explanatory feature for the disproportionately poorer obstetric outcomes seen in African American women compared with women of other races.

Given that SCD is a high-risk condition with increased morbidity and mortality in both pregnant and nonpregnant patients, we hypothesized that pregnancy would pose a physiologic stress resulting in adverse outcomes in a group with SCT compared with women with normal hemoglobin profiles. Though vaso-occlusive polymers are not consistently found in the peripheral blood in patients with SCT, hematologic alterations have been demonstrated to occur when red blood cells are in altered physiologic environments including a shift in $\mathrm{pH}$, oxygen tension, and solubility. Thus, SCT is reliably linked to hematuria, exercise-related sudden death, exertional rhabdomyolysis, and renal papillary necrosis. ${ }^{6}$ However, SCT was not associated with the obstetric outcomes examined in our study, even under the cardiovascular strain that can occur during pregnancy.

Our study is an important contribution to the literature on a condition that disproportionately affects African American women. At a time when more statistics are being exposed about the longstanding obstetric disparities that African American women endure, our results can aid with prenatal counseling in this medically vulnerable patient population and provide reassurance that SCT does not appear to be an independent contributor to the aforementioned adverse outcomes. 
To better target subpopulations that would benefit from increased antenatal surveillance, future research could investigate whether women with concurrent hypertensive disorders and SCT are at higher risk for adverse health outcomes compared with women with either entity alone. In addition, investigation into why women of racial/ethnic minorities have a disproportionately high incidence of $\mathrm{PIH}$, SGA infants, GDM, and PTD is crucial in our understanding and eventual elimination of racial disparities in reproductive health outcomes.

The study has several strengths and limitations. The use of electronic medical records allowed for a thorough collection of information with little missing data. Furthermore, restricting our study population to women solely with SCT or normal Hb profiles with no evidence of hereditary hemolytic anemia conditions obviated confounding by other hemoglobinopathies. We used conservative values in our power calculation to avoid a type II error. Given our large and racially/ethnically diverse sample, the data are generalizable to a variety of practice settings and patient populations. The retrospective study design is a primary limitation of this study. There is also potential for ascertainment bias. Screening is ethnicity-based, which is self-reported by patients. Therefore, those who selfidentify as African American and/or Asian Pacific Islander are more likely to be recommended for hemoglobin electrophoresis than those who do not undergo screening.

\section{Conclusions}

SCT does not appear to be independently associated with increased risk of adverse pregnancy outcomes of PIH, SGA, GDM, and PTD. The findings from this study may inform counseling of patients who screen positive for SCT. Our findings support previous data that race/ethnicity is a risk factor for adverse pregnancy outcomes.
Conflicts of Interest

The authors report no conflicts of interest.

Acknowledgments

We acknowledge Isabel Lazo, MD, ${ }^{\text {a }}$ for her presentation of the ePoster at ACOG in 2016.

\section{References}

1 Bryant AS, Worjoloh A, Caughey AB, Washington AE. Racial/ethnic disparities in obstetric outcomes and care: prevalence and determinants. Am J Obstet Gynecol 2010;202(04):335-343

2 Oteng-Ntim E, Meeks D, Seed PT, et al. Adverse maternal and perinatal outcomes in pregnant women with sickle cell disease: systematic review and meta-analysis. Blood 2015;125(21): 3316-3325

3 Tita AT, Biggio JR, Chapman V, Neely C, Rouse DJ. Perinatal and maternal outcomes in women with sickle or hemoglobin $C$ trait. Obstet Gynecol 2007;110(05):1113-1119

4 Stamilio DM, Sehdev HM, Macones GA. Pregnant women with the sickle cell trait are not at increased risk for developing preeclampsia. Am J Perinatol 2003;20(01):41-48

5 Eichner ER. Sickle cell considerations in athletes. Clin Sports Med 2011;30(03):537-549

6 Tsaras G, Owusu-Ansah A, Boateng FO, Amoateng-Adjepong Y. Complications associated with sickle cell trait: a brief narrative review. Am J Med 2009;122(06):507-512

7 Larrabee KD, Monga M. Women with sickle cell trait are at increased risk for preeclampsia. Am J Obstet Gynecol 1997;177 (02):425-428

8 Gordon NP. Similarity of the adult Kaiser Permanente membership in Northern California to the insured and general population in Northern California: statistics from the 2011-12 California Health Interview Survey. Oakland, CA: Kaiser Permanente Division of Research. http://www.dor.kaiser.org/external/chis_ non_kp_2011. Published June 2015. Accesed February 1, 2017

9 Carpenter MW, Coustan DR. Criteria for screening tests for gestational diabetes. Am J Obstet Gynecol 1982;144(07):768-773

10 Whalley PJ, Pritchard JA, Richards JR Jr. Sickle cell trait and pregnancy. JAMA 1963;186:1132-1135

\footnotetext{
a University of Chicago/NorthShore University Health Systems, Department of Gynecology Oncology.
}

Erratum: The article has been corrected as per Erratum published on January 14, 2020. DOI of the Erratum is 10.1055/s-0040-1701199. 